\title{
CT Scanning in Suspected Stroke or Head Trauma: Is it Worth Going the Extra Mile and Including the Chest to Screen for COVID-19 Infection?
}

\author{
(D) R.M. Kwee, (D). Krdzalic, (DB.A.C.M. Fasen, and (D)T.M.H. de Jaegere, \\ for the COVID-19 CT Investigators South-East Netherlands (CISEN) Study Group
}

\begin{abstract}
BACKGROUND AND PURPOSE: Chest CT may be used as a tool for rapid coronavirus disease 2019 (COVID-19) detection. Our aim was to investigate the value of additional chest CT for detection of coronavirus 19 (COVID-19) in patients who undergo head CT for suspected stroke or head trauma in a COVID-19-endemic region.
\end{abstract}

MATERIALS AND METHODS: Our study included 27 patients (mean age, 74 years; range, 54-90 years; 20 men) who underwent head CT for suspected stroke $(n=21)$ or head trauma $(n=6)$, additional chest CT for COVID-19 detection, and real-time reverse transcriptase polymerase chain reaction testing in a COVID-19-endemic region. Sensitivity, specificity, and negative and positive predictive values of chest CT in detecting COVID-19 were calculated.

RESULTS: Final neurologic diagnoses were ischemic stroke $(n=11)$, brain contusion $(n=5)$, nontraumatic intracranial hemorrhage $(n=2)$, brain metastasis $(n=1)$, and no primary neurologic disorder $(n=8)$. Symptoms of possible COVID-19 infection (ie, fever, cough, and/or shortness of breath) were present in 20 of 27 (74\%) patients. Seven of 27 patients (26\%) had real-time reverse transcriptase polymerase chain reaction confirmed-COVID-19 infection. Chest CT results were 6 true-positives, 15 true-negatives, 5 false-positives, and 1 false-negative. Diagnostic performance values of chest CT were a sensitivity of $85.7 \%$, specificity of $75.0 \%$, negative predictive value of $93.8 \%$, and positive predictive value of $54.6 \%$.

CONCLUSIONS: The sensitivity of additional chest CT is fairly high. However, a negative result does not exclude COVID-19. The positive predictive value is poor. Correlation of chest CT results with epidemiologic history and clinical presentation, along with real-time reverse transcriptase polymerase chain reaction, is needed for confirmation.

ABBREVIATIONS: COVID-19 = coronavirus disease 2019; RT-PCR = real-time reverse transcriptase polymerase chain reaction

T he coronavirus disease 2019 (COVID-19) pandemic has caused a global crisis. As of April 19, there were 2,356,475 confirmed cases and 162,070 deaths worldwide. ${ }^{1}$ In regions where COVID-19 is highly endemic, some hospitals can barely handle the influx of patients. ${ }^{2-4}$ Nevertheless, rapid triage and isolation of patients with suspected COVID-19 are essential to prevent hospital-related transmission of the virus to other patients and health care workers. ${ }^{5,6}$ Stroke and traumatic brain injury are highly prevalent worldwide ${ }^{7}$ and remain common reasons for emergency department visits. ${ }^{8}$ These patients should be assessed for COVID-19 infection on emergency department arrival in

Received April 20, 2020; accepted after revision April 29

From the Department of Radiology (R.M.K., J.K., B.A.C.M.F., T.M.H.d.J.), Zuyderland Medical Center, Heerlen/Sittard/Geleen, the Netherlands.

Please address correspondence to Robert M. Kwee, MD, PhD, Department of Radiology, Zuyderland Medical Center, Henri Dunantstraat 5, 6419 PC Heerlen, the Netherlands; e-mail: rmkwee@gmail.com

- Indicates open access to non-subscribers at www.ajnr.org

http://dx.doi.org/10.3174/ajnr.A6607
COVID-19-endemic areas. One possible strategy is to perform real-time reverse transcriptase polymerase chain reaction (RTPCR) in patients who are being hospitalized. However, a major disadvantage of RT-PCR testing is that it takes several hours to obtain results. It has been suggested that chest CT may be used as a tool for rapid COVID-19 detection in endemic areas. ${ }^{9}$ Head CT is the primary imaging technique of choice to evaluate patients with suspected stroke ${ }^{10,11}$ or head trauma ${ }^{12}$ in the emergency setting. Thus, it may be possible to perform additional chest CT for COVID-19 detection in 1 session with head CT. This approach could be particularly useful when rapid triaging is needed and RT-PCR testing capacity and the number of isolation rooms are limited. Furthermore, such a strategy poses a negligible extra burden on available CT equipment and radiology personnel. In addition, the additional radiation exposure of chest CT is not a major concern because patients with suspected stroke or head trauma are usually older and chest CT can be performed with a low-dose protocol. ${ }^{13}$ Therefore, the purpose of our study was to investigate 


\begin{tabular}{|c|c|c|}
\hline $\begin{array}{l}\text { COVID-19 Pneumonia } \\
\text { Imaging Classification }\end{array}$ & Rationale & CT Findings \\
\hline Typical appearance & $\begin{array}{l}\text { Commonly reported imaging features } \\
\text { of greater specificity for COVID-19 } \\
\text { pneumonia }\end{array}$ & $\begin{array}{l}\text { Peripheral, bilateral GGOs with or without consolidation or visible } \\
\text { intralobular lines ("crazy-paving") } \\
\text { Multifocal GGOs of rounded morphology with or without } \\
\text { consolidation or visible intralobular lines (crazy-paving) } \\
\text { Reverse halo sign or other findings of organizing pneumonia (seen } \\
\text { later in the disease) }\end{array}$ \\
\hline $\begin{array}{l}\text { Indeterminate } \\
\text { appearance }\end{array}$ & $\begin{array}{l}\text { Nonspecific imaging features of } \\
\text { COVID-19 pneumonia }\end{array}$ & $\begin{array}{l}\text { Absence of typical features AND } \\
\text { Presence of: } \\
\text { Multifocal, diffuse, perihilar, or unilateral GGO with or without } \\
\text { consolidation lacking a specific distribution and are non-rounded or } \\
\text { non-peripheral } \\
\text { Few very small GGO with a non-rounded and non-peripheral } \\
\text { distribution }\end{array}$ \\
\hline Atypical appearance & $\begin{array}{l}\text { Uncommonly or not reported } \\
\text { features of COVID-19 pneumonia }\end{array}$ & $\begin{array}{l}\text { Absence of typical or indeterminate features AND } \\
\text { Presence of: } \\
\text { Isolated lobar or segmental consolidation without GGO } \\
\text { Discrete small nodules (centrilobular, "tree-in-bud") } \\
\text { Lung cavitation } \\
\text { Smooth interlobular septal thickening with pleural effusion }\end{array}$ \\
\hline Negative for pneumonia & No features of pneumonia & No CT features to suggest pneumonia \\
\hline
\end{tabular}

Note:-GGOs indicates ground-glass opacities

the value of additional chest CT for COVID-19 detection in patients who undergo head CT for suspected stroke or head trauma in a COVID-19-endemic region.

\section{MATERIALS AND METHODS \\ Patients}

This retrospective study was approved by the institutional review board of our hospital (Zuyderland Medical Center, Heerlen/ Sittard/Geleen, the Netherlands) (No. Z2020061), and patient consent was waived. We included 27 consecutive patients (mean age, 74 years; range, 54-90 years; 20 men) who were evaluated for suspected stroke $(n=21)$ or head trauma $(n=6)$ in our hospital between March 12, 2020, and April 14, 2020. Our hospital, one of the largest general hospitals in the Netherlands, was located in a COVID-19-endemic region. Our study period encompassed the peak of the highest registered number of daily COVID-19 infections in the Netherlands. ${ }^{14}$ During the study period, patients had to be transported to other hospitals because the intensive care unit of our hospital was becoming overloaded (as of April 1, 2020, eighty-three percent of all intensive care unit beds were occupied by patients with COVID-19). All 27 patients underwent head CT, additional chest CT for COVID-19 detection, and RTPCR testing of nasopharyngeal and oropharyngeal swabs. In 3 patients with suspected stroke, CTA was also performed from the aortic arch to the vertex to evaluate for intracranial large-vessel occlusion.

\section{Chest CT Protocol}

Chest CT was performed on either a 64-section CT scanner (Incisive; Philips Healthcare) or on a 64-section dual-source scanner (Somatom Definition Flash; Siemens). Scanning parameters were the following: collimation $=64 \times 0.625$ or $0.6 \mathrm{~mm}$, Tube voltage $=120 \mathrm{kV}$ (peak), Tube current $=667$ maximum $\mathrm{mA}$ or 404 maximum $\mathrm{mA}$, pitch $=1.0$ or 1.2 , and matrix size $5=$
$12 \times 512$. CT images were reconstructed with a lung kernel in the transverse plane with a $1.0-\mathrm{mm}$ section thickness and a $1.0-\mathrm{mm}$ increment. Images were also reconstructed in axial, coronal, and sagittal planes with a $3.0-\mathrm{mm}$ section thickness.

\section{Chest CT Interpretation and Reference Standard}

Chest CT findings were categorized according to the Radiological Society of North America chest CT classification system for reporting COVID-19 pneumonia-ie, typical, indeterminate, atypical, and negative (Table 1). ${ }^{15}$ Typical and indeterminate chest CT findings were considered positive, whereas atypical and negative chest CT findings were considered negative for COVID19. All chest CT scans were prospectively read by radiologists who were available when CT was performed. Results of RT-PCR testing, (ie, positive or negative for COVID-19 infection) served as the reference standard.

\section{Statistical Analysis}

The number of true-positive, true-negative, false-positive, and true-negative chest CT results was determined. Subsequently, sensitivity, specificity, negative predictive value, and positive predictive value of chest CT in diagnosing COVID-19 were calculated. Statistical analyses were performed using SPSS Statistics for Windows (Version 20.0; IBM).

\section{RESULTS}

\section{Patients}

Final neurologic diagnoses were ischemic stroke $(n=11)$, brain contusion $(n=5)$, nontraumatic intracranial hemorrhage $(n=$ $2)$, brain metastasis $(n=1)$, and no primary neurologic disorder $(n=8)$. Correlation of symptoms of possible COVID-19 infection (ie, fever, cough, and/or shortness of breath ${ }^{16}$ ), chest CT findings, and RT-PCR results are shown in Table 2. Symptoms of possible COVID-19 infection were present in 20 of 27 (74\%) 
patients. The median time interval between CT and RT-PCR was 0 days (range, 0-7, days; interquartile range, 0-1 day). In 2 of 4 patients with a chest CT result positive for COVID-19 but negative findings on the initial RT-PCR, repeat RT-PCR was performed (with findings remaining negative). Seven of 27 patients (26\%) had RT-PCR-confirmed COVID-19 infection. Six patients

Table 2: Correlation between symptoms of possible COVID-19 infection (ie, fever, cough, and/or shortness of breath ${ }^{16}$ ), chest CT findings, and RT-PCR results

\begin{tabular}{lcc}
\hline & $\begin{array}{c}\text { RT-PCR, } \\
\text { Positive } \\
\text { Results }\end{array}$ & $\begin{array}{c}\text { RT-PCR, } \\
\text { Negative } \\
\text { Results }\end{array}$ \\
\hline Chest CT, positive findings & $5^{\mathrm{a}} / 1^{\mathrm{b}}$ & $3^{\mathrm{a}} / 2^{\mathrm{b}}$ \\
Chest CT, negative findings & $1^{\mathrm{a}} / 0^{\mathrm{b}}$ & $11^{\mathrm{a}} / 4^{\mathrm{b}}$ \\
\hline
\end{tabular}

a Symptomatic patients.

${ }^{\mathrm{b}}$ Asymptomatic patients.

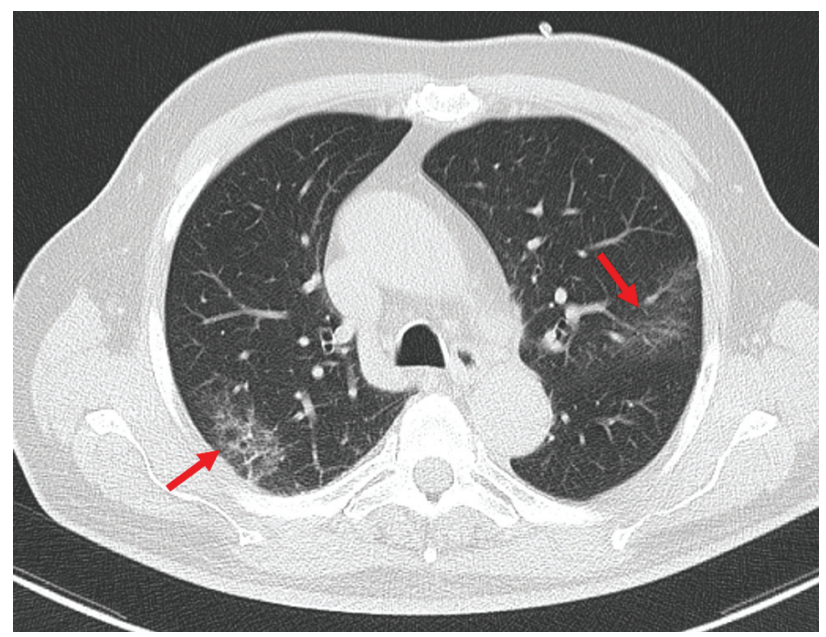

FIG 1. A 73-year-old male patient who underwent head CT and CTA to evaluate intracranial large-vessel occlusion. This patient did not have symptoms of COVID-19 infection (ie, no fever, cough, and/or shortness of breath). Axial chest CT image shows ground-glass opacities posteriorly located in both upper lobes (arrows). These CT features were interpreted as typical for COVID-19 pneumonia, which was confirmed by RT-PCR.
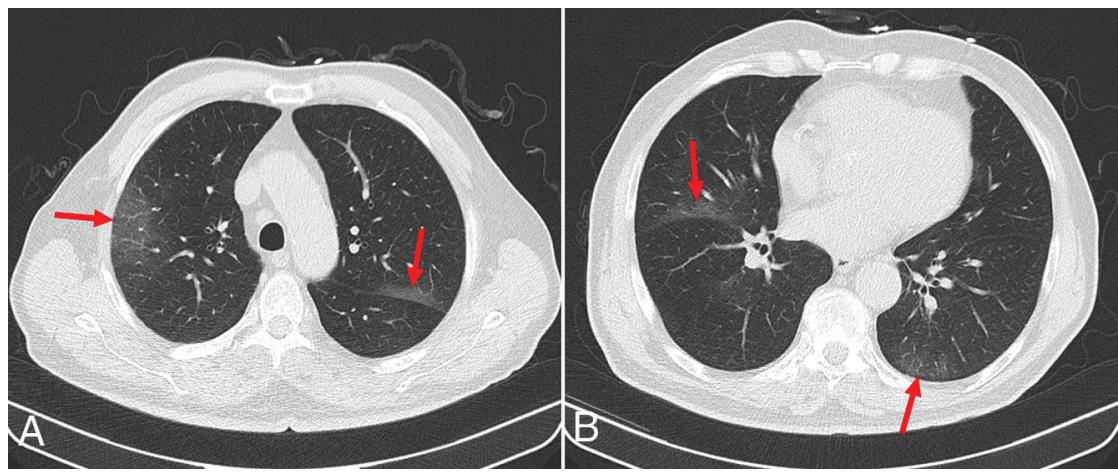

FIG 2. An 83-year-old male patient who underwent head CT for suspected stroke. This patient had symptoms of possible COVID-19 infection (cough and shortness of breath). Axial chest CT images at the level of the aortic arch $(A)$ and heart $(B)$ show ground-glass opacities posteriorly and peripherally, located in both upper lobes (arrows in $A$ and $B$ ) and in the left lower lobe (arrow, $B$ ). These CT features were interpreted as typical for COVID-19 pneumonia, which was confirmed by RT-PCR. with RT-PCR-confirmed COVID-19 were symptomatic, whereas 1 patient with RT-PCR-confirmed COVID-19 was asymptomatic. In the asymptomatic patient with RT-PCR-confirmed COVID-19, abnormalities suspicious for COVID-19 were already visible in the upper lung fields on CTA, which was confirmed on additional chest CT (Fig 1). In another patient with head trauma and fever, chest CT findings were negative, whereas RT-PCR 4 days after CT showed COVID-19. This patient was not isolated until the RT-PCR results were known.

\section{Diagnostic Yield of Chest CT}

Typical, indeterminate, atypical, and negative chest CT features for COVID-19 were present in 3, 8, 2, and 14 patients, respectively. Figs 1 and 2 show examples of typical chest CT features of COVID-19, whereas Fig 3 shows an example of atypical chest CT features of COVID-19. Chest CT results were 6 true-positives, 15 true-negatives, 5 false-positives, and 1 false-negative. Diagnostic performance values of chest CT were a sensitivity of $85.7 \%$, specificity of $75.0 \%$, negative predictive value of $93.8 \%$, and positive predictive value of $54.6 \%$.

\section{DISCUSSION}

Our initial experience in 27 patients with an emergency indication for head CT shows that the sensitivity of additional chest CT for COVID-19 is fairly high. An estimated 5\%-80\% of COVID-19-infected persons may be asymptomatic. ${ }^{17}$ Although asymptomatic, they may transmit the disease to others. ${ }^{18-21}$ In our study, 7 of 27 patients (26\%) did not have symptoms of possible COVID-19 infection (ie, fever, cough, and/or shortness of breath ${ }^{16}$ ). One of these asymptomatic patients proved to be infected with COVID-19 by RT-PCR. This finding underlines the importance of vigilantly evaluating the lungs on CTA scans of patients with stroke in a COVID-19-endemic region, even when they are asymptomatic. However, a negative result does not exclude COVID-19. In 1 patient with head trauma and fever, chest CT had negative findings, whereas RT-PCR 4 days after CT showed COVID-19 infection. This patient was not isolated until the RT-PCR results were known and could have put other patients and health care workers at risk to become infected too. Furthermore, the positive predictive value of chest CT was poor. If only chest CT results were used to make patientmanagement decisions, too many cases would unnecessarily occupy isolation rooms. Therefore, correlation with epidemiologic history and clinical presentation, along with the RT-PCR, is needed for confirmation. $^{22}$

The diagnostic accuracy of chest CT has been investigated by a few previous studies, ${ }^{9,23,24}$ in different populations. Our study is unique in that it investigated the value of additional chest CT in patients who already underwent head CT in a COVID-19endemic region, compared with 


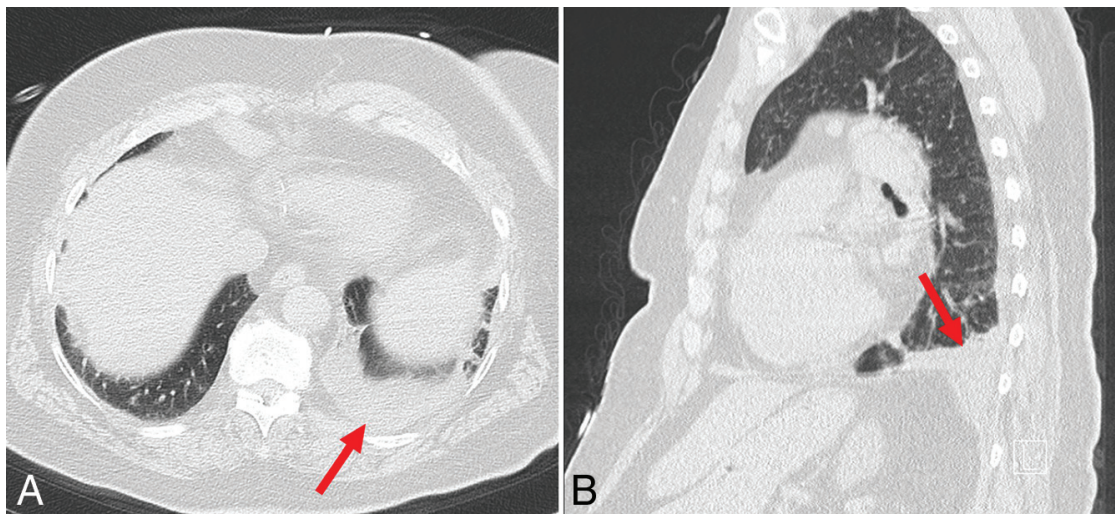

FIG 3. An 83-year-old female patient who underwent head CT for suspected stroke. This patient had symptoms of possible COVID-19 infection (shortness of breath). Axial (A) and sagittal (B) chest CT images show segmental consolidation in the left lower lobe (arrows, $A$ and $B$ ) and no other long abnormalities. These $C T$ features were interpreted as atypical for COVID-19 pneumonia. RT-PCR was negative for COVID-19.

previous studies that reported chest CT as a stand-alone method in patients who may otherwise not have undergone CT scanning. ${ }^{9,23,24}$ Furthermore, our study also included asymptomatic patients (26\% of our study population) with respect to possible COVID-19. Because of the inclusion of patients without any COVID-19 symptoms, the overall prevalence of COVID-19 infection in our study population was lower than that in previous studies ${ }^{9,23,24}$ (26\% in our study versus $39 \%-$ $85 \%$ in previous studies ${ }^{9,23,24}$ ). Nevertheless, the fairly high sensitivity and poor specificity of additional chest CT is in line with the findings of previous studies that reported sensitivity and specificity values of $93.2 \%-97.0 \%$, and $25.0 \%-53.3 \%$, respectively. ${ }^{9,23,24}$ Most important, our observation that a negative chest CT result does not exclude COVID-19 is supported by a study among passengers from the cruise ship Diamond Princess, which showed that 6 of 28 symptomatic patients with COVID-19 had normal chest CT findings. ${ }^{25}$

RT-PCR of nasopharyngeal and oropharyngeal specimens is considered the standard to diagnose COVID-19. ${ }^{26-29}$ However, currently available RT-PCR tests are time-consuming (it takes several hours before the results are known), and there is still relatively limited RT-PCR testing capacity worldwide. ${ }^{30,31}$ In a heavily constrained environment with a lack of sufficient RT-PCR testing capacity and a shortage of isolation beds, additional chest CT may be performed to detect COVID-19 in patients who have already undergone head CT. The data from this study may be used to weigh the potential advantages and disadvantages of performing additional chest $\mathrm{CT}$ in such settings. On a different note, additional chest CT in patients who require CT scanning of other body regions may also be helpful for taking appropriate actions in a timely manner to maintain the safety of the CT room. If chest CT findings are positive for possible COVID-19, the CT room should be decontaminated (room downtime for decontamination is typically between 30 minutes to 1 hour $^{32}$ ) before scanning the next patient.

Our study has some limitations. First, the number of included patients was relatively low. Nevertheless, we were able to demonstrate the value of additional chest CT for COVID-19 detection in patients with suspected stroke or head trauma. Second, in needed for confirmation.

only 2 of 5 patients with positive findings on chest CT but negative findings on the initial RT-PCR was repeat RT-PCR performed (with findings remaining negative) due to the limited availability of RT-PCR kits in our hospital. Only patients in whom clinical suspicion of COVID-19 persisted were retested.

\section{CONCLUSIONS}

The sensitivity of additional chest CT is fairly high. However, a negative result does not exclude COVID-19. The positive predictive value is poor. Correlation of chest CT results with epidemiologic history and clinical presentation, along with RT-PCR, is

\section{REFERENCES}

1. COVID-19 Dashboard by the Center for Systems Science and Engineering (CSSE) at Johns Hopkins University (JHU). https:// gisanddata.maps.arcgis.com/apps/opsdashboard/index.html\#/ bda7594740fd40299423467b48e9ecf6. Accessed April 19, 2020

2. Italy's Health Care System Groans Under Coronavirus-a Warning to the World. The New York Times. March 27, 2020. https://www.nytimes.com/2020/03/12/world/europe/12italy-coronavirushealth-care.html. Accessed April 19, 2020

3. Marquez M, Moghe S. Inside a Brooklyn hospital that is overwhelmed with Covid-19 patients and deaths. CNN. March 31, 2020. https://edition.cnn.com/2020/03/30/us/brooklyn-hospital-coronaviruspatients-deaths/index.html. Accessed April 19, 2020

4. Rothfeld M. 13 Deaths in a Day: An 'Apocalyptic' Coronavirus Surge at an N.Y.C. Hospital. The New York Times. March 24, 2020. https://www.nytimes.com/2020/03/25/nyregion/nyc-coronavirushospitals.html. Accessed April 19, 2020

5. Centers for Disease Control and Prevention Interim Infection Prevention and Control Recommendations for Patients with Suspected or Confirmed Coronavirus Disease 2019 (COVID-19) in Healthcare Settings. https://www.cdc.gov/coronavirus/2019-ncov/ hcp/infection-control-recommendations.html. Accessed April 19, 2020

6. Wang D, Hu B, Hu C, et al. Clinical characteristics of 138 hospitalized patients with 2019 novel coronavirus-infected pneumonia in Wuhan, China. JAMA 2020 Feb 7. [Epub ahead of print] CrossRef Medline

7. World Health Organization. Neurological Disorders. https://www. who.int/mental_health/neur. Accessed April 19, 2020

8. Centers for Disease Control and Prevention. Emergency Department Visits. https://www.cdc.gov/nchs/fastats/emergency-department.htm. Accessed April 19, 2020

9. Ai T, Yang Z, Hou H, et al. Correlation of chest CT and RT-PCR testing in coronavirus disease 2019 (COVID-19) in China: a report of 1014 cases. Radiology 2020 Feb 26. [Epub ahead of print] CrossRef Medline

10. Kamalian S, Lev MH. Stroke imaging. Radiol Clin North Am 2019;57:717-32 CrossRef Medline

11. Salmela MB, Mortazavi S, Jagadeesan BD, et al; Expert Panel on Neurologic Imaging. ACR Appropriateness Criteria ${ }^{\circledR}$ Cerebrovascular Disease. J Am Coll Radiology 2017;14:S34-61 CrossRef Medline 
12. Shetty VS, Reis MN, Aulino JM, et al. ACR Appropriateness Criteria head trauma. J Am Coll Radiology 2016;13:668-79 CrossRef Medline

13. Kang Z, Li X, Zhou S. Recommendation of low-dose CT in the detection and management of COVID-2019. Eur Radiol 2020 March 19. [Epub ahead of print] CrossRef Medline

14. National Institute for Public Health and the Environment. Ontwikkeling COVID-19 in grafieken. https:/www.rivm.nl/coronaviruscovid-19/grafieken. Accessed April 19, 2020

15. Simpson S, Kay FU, Abbara S, et al. Radiological Society of North America Expert Consensus Statement on Reporting Chest CT Findings Related to COVID-19: endorsed by the Society of Thoracic Radiology, the American College of Radiology, and RSNA. Radiology: Cardiothoracic Imaging 2020;2:e200152 CrossRef

16. Centers for Disease Control and Prevention. Coronavirus Disease 2019 (COVID-19): Symptoms of Coronavirus. https://www.cdc. gov/coronavirus/2019-ncov/symptoms-testing/symptoms.html. Accessed April 19, 2020

17. Centre for Evidence-Based Medicine. COVID-19: What proportion are asymptomatic? https://www.cebm.net/covid-19/covid-19-whatproportion-are-asymptomatic/. Accessed April 19, 2020

18. Bai Y, Yao L, Wei T, et al. Presumed asymptomatic carrier transmission of COVID-19. JAMA $2020 \mathrm{Feb} 21$. [Epub ahead of print] CrossRef Medline

19. Hu Z, Song C, Xu C, et al. Clinical characteristics of 24 asymptomatic infections with COVID-19 screened among close contacts in Nanjing, China. Sci China Life Sci 2020;63:706-11 CrossRef Medline

20. Yu X, Yang R. COVID-19 transmission through asymptomatic carriers is a challenge to containment. https://onlinelibrary.wiley.com/ doi/pdf/10.1111/irv.12743. Accessed April 19, 2020

21. Rothe C, Schunk M, Sothmann P, et al. Transmission of 2019-nCoV infection from an asymptomatic contact in Germany. $N$ Engl J Med 2020;382:970-71 CrossRef Medline

22. Novel Coronavirus Pneumonia Diagnosis and Treatment Plan (Provisional 7th Edition). http://www.gov.cn/zhengce/zhengceku/ 2020-03/04/5486705/files/ae61004f930d47598711a0d4cbf874a9.pdf. Accessed April 19, 2020
23. Caruso D, Zerunian M, Polici M, et al. Chest CT features of COVID-19 in Rome, Italy. Radiology 2020 April 3. [Epub ahead of print] CrossRef Medline

24. Wen Z, Chi Y, Zhang L, et al. Coronavirus disease 2019: initial detection on chest CT in a retrospective multicenter study of 103 Chinese subjects. Radiology: Cardiothoracic Imaging 2020;2:e200092 CrossRef

25. Inui S, Fujikawa A, Jitsu M, et al. Erratum: chest CT findings in cases from the cruise ship "Diamond Princess" with coronavirus disease 2019 (COVID-19). Radiology: Cardiothoracic Imaging 2020; 2:e204002 CrossRef

26. Huang C, Wang Y, Li X, et al. Clinical features of patients infected with 2019 novel coronavirus in Wuhan, China. Lancet 2020;395: 497-506 CrossRef Medline

27. Guan WJ, Ni ZY, Hu Y, et al; China Medical Treatment Expert Group for Covid-19. Clinical characteristics of coronavirus disease 2019 in China. N Engl J Med 2020;382:1708-20 CrossRef Medline

28. World Health Organization. WHO interim guidance for laboratory testing. https://www.who.int/emergencies/diseases/novel-coronavirus2019/technical-guidance/laboratory-guidance. Accessed April 19, 2020

29. Corman VM, Landt O, Kaiser M, et al. Detection of 2019 novel coronavirus (2019-nCoV) by real-time RT-PCR. Euro Surveill 2020;25 CrossRef Medline

30. Cheng MP, Papenburg J, Desjardins M, et al. Diagnostic testing for severe acute respiratory syndrome-related coronavirus-2: a narrative review. Ann Intern Med 2020 April 13. [Epub ahead of print] CrossRef Medline

31. National Institute for Public Health and the Environment. Policy on testing for novel coronavirus disease (COVID-19). https://www. rivm.nl/en/novel-coronavirus-covid-19/what-are-we-doing-in-thenetherlands-in-response-to-the-coronavirus/testing-policy. Accessed April 19, 2020

32. Mossa-Basha M, Meltzer CC, Kim DC, et al. Radiology department preparedness for COVID-19: Radiology Scientific Expert Panel. Radiology 2020 Mar 16. [Epub ahead of print] CrossRef Medline 